\title{
A reconciliation approach to key generation based on Module-LWE
}

\author{
Charbel Saliba and Laura Luzzi \\ ETIS, UMR 8051, \\ CY Université, ENSEA, CNRS, \\ Cergy, France \\ Email: \{charbel.saliba, laura.luzzi\}@ensea.fr
}

\author{
Cong Ling \\ Department of Electrical \\ and Electronic Engineering \\ Imperial College London, U.K. \\ Email: cling@ieee.org
}

\begin{abstract}
We consider a key encapsulation mechanism (KEM) based on Module-LWE where reconciliation is performed on the 8-dimensional lattice $E_{8}$, which admits a fast CVP algorithm. Our scheme generates 256 bits of key and requires 3 or 4 bits of reconciliation per dimension. We show that it can outperform Kyber in terms of the modulus $q$ with comparable error probability. We prove that our protocol is IND-CPA secure and improves the security level of Kyber by $7.3 \%$.
\end{abstract}

\section{INTRODUCTION}

Over the past few years, there have been many attractive developments in lattice-based cryptographic protocols, whose security is based on worst-case hardness assumptions, and which are conjectured to be secure against quantum attacks. Thus, lattice-based primitives are a promising candidate to replace constructions based on number theoretic assumptions like RSA [1] or Diffie-Hellman [2] that are currently in use.

One of the most versatile primitives for the design of provably secure cryptographic protocols is the learning with errors (LWE) problem introduced by Regev [3]. For instance it can serve for IND-CPA (Indistinguishability under chosenplaintext attack) [3] and IND-CCA (Indistinguishability under chosen-ciphertext attack) public key encryption [4]. A structured variant of LWE, the decision ring learning with errors ( $R$-LWE) was proposed in [5] by Lyubashevsky et al. to allow more compact representations, which in turn inspired the authors of [6] to introduce the module learning with errors ( $M$-LWE) variant. This new problem seems to offer better security guarantees and more flexibility in designing cryptographic schemes.

In [7], Peikert introduced an efficient lattice-based key encapsulation mechanism (KEM) based on $R$-LWE that allows two parties to share an ephemeral key that is useful for secret communications. Peikert's scheme features a low bandwidth reconciliation technique that aims to reach exact agreement on the shared key. A practical implementation of Peikert's protocol called NEWHOPE was proposed in [8] as a candidate to the NIST challenge on post-quantum cryptography. In [7] and [8], although key generation is performed using 1024dimensional lattices, the reconciliation step uses 1-dimensional and 4-dimensional lattices respectively 1 . Subsequently, Kyber

\footnotetext{
${ }^{1}$ In fact, the latest implementation of the NewHope algorithm does not use reconciliation [9].
}

appears as an alternative approach to the NewHope KEM [10, 11]. Based on the hardness of Module-LWE, the authors of [11] construct a IND-CCA secure KEM from a CPA-secure public-key encryption scheme which achieve 165 bits of postquantum security, together with an error probability less than $2^{-164}$ using modulus $q=3329$.

In this paper, we consider a key-generation protocol based on Module-LWE using the reconciliation technique. We consider the cyclotomic ring of degree $n=256$ as in Kyber. Compared to NewHope, we need to use a higher-dimensional lattice for reconciliation in order to generate one bit of key per dimension. We choose the 8-dimensional Gosset lattice $E_{8}$ due to its optimal density and low-complexity quantization.

We show that our scheme can guarantee a smaller error probability than Kyber's, i.e. $P_{e}<2^{-174}$, with a smaller modulus $q=2^{11}$, using 4 bits of reconciliation per dimension. For this choice of $q$, our scheme achieves 176 bits of postquantum security.

A smaller error probability is desirable to prevent leakage of information from decryption failure attacks [12], while a smaller modulus-to-noise ratio provides better efficiency and stronger concrete security against known attacks.

We note that by choosing $q$ to be a prime number as in Kyber, one can use the Number Theoretic Transform to speed up polynomial multiplication [13, 7, 8]. However, prime $q$ is not required for security [14], and power-of-two moduli have been used in the literature in [15, 16]. These works use other methods for efficient polynomial multiplication, e.g. Karatsuba / Toom-Cook algorithms and index-based multiplication. An advantage of choosing an even $q$ is that a dither is not required to obtain a uniform key, unlike [7, 8]. We note that the hardness of Module-LWE has been established for general modulus $q[17$.

Organization: This paper is organized as follows. In Section III we provide basic definitions about cyclotomic fields, lattices, etc. In Section III, we introduce our key generation algorithm. In Section [V] and $\mathrm{V}$, we provide a proof that the error probability is small, and that our scheme is IND-CPA secure respectively. Finally, in Section VI we provide estimates for concrete security against known attacks. 


\section{Preliminaries}

In this section, we introduce the mathematical tools we use to describe and analyze our proposed scheme.

\section{A. Lattices and Algebraic number theory}

Lattice definitions: For our purposes, a lattice $\Lambda$ is a real full-rank discrete additive subgroup of $\mathbb{R}^{n}$. Any lattice is generated as the set of all integer linear combinations of $n$ linearly independent basis vectors $\mathbf{B}=\left\{\mathbf{b}_{1}, \ldots, \mathbf{b}_{n}\right\}$ in $\mathbb{R}^{n}$. The Voronoi region of $\Lambda$, denoted $\mathcal{V}(\Lambda)$ is the set of all points in $\mathbb{R}^{n}$ which are closest to the origin than to any other lattice point. A Voronoi-relevant vector is an element $\lambda \in \Lambda$ such that $\langle\mathbf{x}, \lambda\rangle<\|\mathbf{x}\|^{2}$ for all $\mathbf{x} \in \Lambda \backslash\{0, \lambda\}$. For any $\mathbf{x} \in \mathbb{R}^{n}$, we denote by $Q_{\Lambda}(\mathbf{x})$ the closest vector in $\Lambda$ to $\mathbf{x}$. We also define the modulo $\Lambda$ operation as $\mathbf{x} \bmod \Lambda=\left(\mathbf{x}-Q_{\Lambda}(\mathbf{x})\right) \in \mathcal{V}(\Lambda)$. We will use implicitly in our proofs the fact that $\forall \mathbf{x}, \mathbf{y} \in \mathbb{R}^{n}$, and $\forall \lambda \in \Lambda,(\mathbf{x} \bmod \Lambda+\mathbf{y}) \bmod \Lambda=(\mathbf{x}+\mathbf{y}) \bmod \Lambda$ as well as $(\mathbf{x}+\lambda) \bmod \Lambda=\mathbf{x} \bmod \Lambda$.

Lemma 1. Let $\Lambda^{\prime} \subset \Lambda$ and $\lambda \in \Lambda$; then $\pi: \Lambda / \Lambda^{\prime} \rightarrow \Lambda / \Lambda^{\prime}$ defined as $\pi(v)=(v+\lambda) \bmod \Lambda^{\prime}$ is a permutation of $\Lambda / \Lambda^{\prime}$.

Cyclotomic fields and modules: For a power of 2 integer $m \geq 1$, the $m^{\text {th }}$ cyclotomic number field is the extension $K=$ $\mathbb{Q}\left(\zeta_{m}\right)$ with degree $n=m / 2$, where $\zeta_{m}$ is any $m^{\text {th }}$ primitive root of unity. The ring of integers of $K$ is $R=\mathbb{Z}\left[\zeta_{m}\right] \cong$ $\mathbb{Z}[X] /\left(X^{n}+1\right)$ and given $q>0$, its quotient ring is $R_{q}=$ $\mathbb{Z}_{q}[X] /\left(X^{n}+1\right)$. For an integer $d>0$, a subset $M \subseteq K^{d}$ is an $R$-module if it is closed under addition and multiplication by elements of $R$.

Polynomial splitting: We will use a polynomial splitting in section IV to analyse our error probability bound, similarly to $\left[8\right.$, Section C]. For that, we write $n=n_{0} \times L$ and take $S=\mathbb{Z}[Y] /\left(Y^{n_{0}}+1\right)$.

Given a polynomial $a(X) \in R$, we define for $\kappa=0, \ldots, L-1$ the vectors $a^{(\kappa)}$ as $a^{(\kappa)}=\left(a_{\kappa}, a_{\kappa+L}, a_{\kappa+2 L}, \ldots, a_{\kappa+n-L}\right)$. Taking $Y=X^{L}$ allows to write $a(X)=\sum_{\kappa=0}^{L-1} a^{(\kappa)}(Y) X^{\kappa}$. This in turn allows to express the multiplication $a(X) b(X)$ as $p(X):=a(X) b(X)=\sum_{\kappa=0}^{L-1} p^{(\kappa)}(Y) X^{\kappa}$. Hence,

$$
p^{(\kappa)}(Y)=\sum_{i=0}^{L-1} Y^{\delta_{i, \kappa}} a^{(i)}(Y) \cdot b^{(\kappa-i \bmod L)}(Y),
$$

where $\delta_{i, \kappa}$ is either 0 or 1 . In the sequel we take out the $\bmod L$ operation to simplify notations.

\section{B. E $E_{8}$ lattice}

The $E_{8}$ lattice is a discrete subgroup of $\mathbb{R}^{8}$ of full rank [18, p.121]. One possible basis for $E_{8}$ is given by the rows of the matrix

$$
\mathbf{E}=\left[\begin{array}{cccccccc}
2 & 0 & 0 & 0 & 0 & 0 & 0 & 0 \\
-1 & 1 & 0 & 0 & 0 & 0 & 0 & 0 \\
0 & -1 & 1 & 0 & 0 & 0 & 0 & 0 \\
0 & 0 & -1 & 1 & 0 & 0 & 0 & 0 \\
0 & 0 & 0 & -1 & 1 & 0 & 0 & 0 \\
0 & 0 & 0 & 0 & -1 & 1 & 0 & 0 \\
0 & 0 & 0 & 0 & 0 & -1 & 1 & 0 \\
1 / 2 & 1 / 2 & 1 / 2 & 1 / 2 & 1 / 2 & 1 / 2 & 1 / 2 & 1 / 2
\end{array}\right]
$$

This lattice has 2 types of Voronoi-relevant vectors of the form $\left( \pm 1^{2}, 0^{6}\right) \in \mathrm{VR}_{1}$ and $\left( \pm 0.5^{8}\right) \in \mathrm{VR}_{2}$. Note that $\left|\mathrm{VR}_{1}\right|=112$ and $\left|\mathrm{VR}_{2}\right|=128$, so that the total is 240 . The volume of $E_{8}$ is simply 1 . A simple and fast CVP algorithm for $E_{8}$ is given in [19]. Note that $2 \mathbb{Z}^{8} \subset E_{8} \subset \frac{1}{2} \mathbb{Z}^{8}$.

\section{Gaussian-like Error Distribution}

When dealing with module-LWE defined below, we work with a Gaussian-like error distribution over the ring $R^{d}$.

Since it is challenging to implement a discrete Gaussian sampler which is efficient and protected against timing attacks, one can replace the secret and error distribution by the centered binomial distribution $\psi_{k}$ of standard deviation $\sqrt{k / 2}$ introduced in [8], which is defined as

$$
\psi_{k}=\sum_{i=1}^{k}\left(b_{i}-b_{i}^{\prime}\right)
$$

where $b_{i}, b_{i}^{\prime}$ are independent and uniformly distributed in $\{0,1\}$, for $i=1, \ldots, k$.

Note that choosing $\psi_{k}$ as error distribution does not significantly decrease security compared to a rounded Gaussian distribution, and this can be shown with a Rényi divergencebased analysis, as in [8, Theorem 4.1] for $k=16$. It remains true for a general value of $k$ [20, Section 5.3].

If $x \in R$, we write $x \leftarrow \Psi_{k}$ to mean that $x \in R$ is generated from a distribution where each of its coefficients is generated according to $\psi_{k}$. Similarly, a $d$-dimensional vector $\mathbf{x} \in R^{d}$ can be generated according to the distribution $\Psi_{k}^{d}$.

\section{Cryptography and Ring-LWE}

Cryptographic definitions: We define the notion of key encapsulation mechanism (KEM) following [7], which consists of three algorithms (Gen, Encaps, Decaps), where Gen takes a public parameter $p p$ and returns a secret key $s k$ and a private one $p k$, where Encaps takes $(p p, p k)$ to produce a ciphertext $c$ and a key $\mathbf{k} \in \mathcal{K}$, and where Decaps takes the secret key $s k$ and ciphertext $c$ to return a key $\mathbf{k} \in \mathcal{K}$ or the symbol $\perp$ to denote rejection. A KEM satisfies INDCPA security, if the outputs of the following "real" and "ideal" games are computationally indistinguishable:

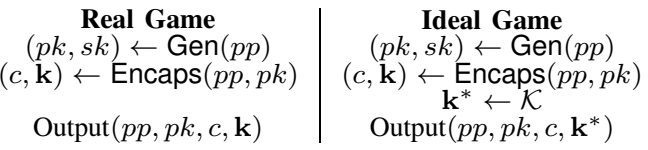

Module-LWE (M-LWE): The security of our scheme is based on the hard module-LWE problem [6]. Let $d$ be a positive integer parameter. The problem consists in distinguishing uniform samples $\left(\mathbf{a}_{i}, b_{i}\right) \leftarrow R_{q}^{d} \times R_{q}$ from samples $\left(\mathbf{a}_{i}, b_{i}\right) \leftarrow$ $R_{q}^{d} \times R_{q}$ where $\mathbf{a}_{i} \leftarrow R_{q}^{d}$ is uniform and $b_{i}=\mathbf{a}_{i} \cdot \mathbf{s}+e_{i}$ with $\mathbf{s} \leftarrow \Psi_{k}^{d}$ common to all samples and $e_{i} \leftarrow \Psi_{k}$ fresh for every sample. The multiplication $\mathbf{a}_{i} \cdot \mathbf{s}$ is a dot product $a_{1} \cdot s_{1}+\cdots+a_{d} \cdot s_{d}$, such that each $a_{i} \cdot s_{i}$ is a polynomial product modulo $\left(X^{n}+1\right)$.

\section{Key GENERATION ALGORITHM}

We give here the key generation algorithm between Alice and Bob. Our protocol makes use of the following lattices of dimension $n=256$ : the quantization lattice $\Lambda_{1}=\left(\frac{q}{2^{p}} E_{8}\right)^{32}$ 


\begin{tabular}{|lll|}
\hline \multicolumn{2}{|c|}{ Parameters: $q=2^{11} ; k=2 ; n=256 ; d=3$} \\
\hline \hline Alice (server) & Bob(Client) \\
$\mathbf{A} \stackrel{\$}{\leftarrow} R_{q}^{d \times d}$ & & $\mathbf{s}^{\prime}, \mathbf{e}^{\prime} \leftarrow \Psi_{k}^{d}, e^{\prime \prime} \leftarrow \Psi_{k}$ \\
$\mathbf{s}, \mathbf{e} \leftarrow \Psi_{k}^{d}$ & $\stackrel{(\mathbf{A}, \mathbf{b})}{\longrightarrow}$ & \\
$\mathbf{b}:=\mathbf{A} \mathbf{s}+\mathbf{e} \in R_{q}^{d}$ & & $\mathbf{u}:=\mathbf{A}^{T} \mathbf{s}^{\prime}+\mathbf{e}^{\prime} \in R_{q}^{d}$ \\
& & $v:=\mathbf{b} \cdot \mathbf{s}^{\prime}+e^{\prime \prime} \in R_{q}$ \\
& $\stackrel{(\mathbf{u}, r)}{ }$ & $r=\operatorname{HelpRec}(v)$ \\
$v^{\prime}:=\mathbf{u} \cdot \mathbf{s} \in R_{q}$ & & $\mathbf{k}=\operatorname{Rec}(v, r)$ \\
$\mathbf{k}=\operatorname{Rec}\left(v^{\prime}, r\right)$ & & \\
\hline
\end{tabular}

TABLE I

Key generation algorithm based on module-LWE

for some integer $p \geq 1$, the coding lattice $\Lambda_{2}=\left(\frac{q}{2} E_{8}\right)^{32}$ and the shaping lattice $\Lambda_{3}=q\left(\mathbb{Z}^{8}\right)^{32}$. This choice implies that $\Lambda_{3} \subseteq \Lambda_{2} \subseteq \Lambda_{1}$. The key rate given by $R_{K}=\frac{1}{n} \log _{2}\left(\frac{\operatorname{Vol}\left(\Lambda_{3}\right)}{\operatorname{Vol}\left(\Lambda_{2}\right)}\right)$ is simply 1 so that the protocol provides 256 bits of key. Furthermore, the reconciliation rate $R_{P}=\frac{1}{n} \log _{2}\left(\frac{\operatorname{Vol}\left(\Lambda_{2}\right)}{\operatorname{Vol}\left(\Lambda_{1}\right)}\right)$ is calculated to be $p-1$.

With regard to Table $\llbracket$ the KEM algorithm consists of taking a random matrix $\mathbf{A}$ from $R_{q}^{d \times d}$ by Alice, and referring it as the public parameter $p p$. Then she chooses $\mathbf{e}, \mathbf{s} \leftarrow \Psi_{k}^{d}$, computes $\mathbf{b}=\mathbf{A} \mathbf{s}+\mathbf{e}$, and outputs a public key $p k=\mathbf{b}$ and a secret key $s k=\mathbf{s}$. When it's Bob's turn, he chooses independent $\mathbf{e}^{\prime}, \mathbf{s}^{\prime} \leftarrow$ $\Psi_{k}^{d}$ and $e^{\prime \prime} \leftarrow \Psi_{k}$, then computes $\mathbf{u}=\mathbf{A}^{T} \mathbf{s}^{\prime}+\mathbf{e}^{\prime} \in R_{q}^{d}$ and $v=\mathbf{b} \cdot \mathbf{s}^{\prime}+e^{\prime \prime} \in R_{q}$. He outputs $c=(\mathbf{u}, r) \in R_{q}^{d} \times \Lambda_{1} / \Lambda_{2}$ with $r=\operatorname{HelpRec}(v):=Q_{\Lambda_{1}}(v) \bmod \Lambda_{2}$ and $\mathbf{k}$ in $\Lambda_{2} / \Lambda_{3}$ such that $\mathbf{k}=\operatorname{Rec}(v, r):=Q_{\Lambda_{2}}(v-r) \bmod \Lambda_{3}$. At the end, Alice computes $v^{\prime}=\mathbf{u} \cdot \mathbf{s}$, and outputs $\hat{\mathbf{k}}=\operatorname{Rec}\left(v^{\prime}, r\right)$.

Note that when $v \in R_{q}$, the notation $Q_{\Lambda_{1}}(v)$ means that we perform $Q_{\frac{q}{2^{p}} E_{8}}$ on each component $v^{(\kappa)}$ (see Section II) where $n_{0}=8$ and $L=32$, and similarly for $Q_{\Lambda_{2}}(\cdot), \bmod \Lambda_{2}$ and $\bmod \Lambda_{3}$ operations.

Remark 1 (Comparison with NewHope and Kyber [8, 10, 11])). Note that this algorithm is reconciliation-based, similarly to [7] and the first version of the NewHope protocol [8]. For instance, in [8] the functions HelpRec and Rec can be written as the above form by taking the product lattices $\Lambda_{1}=\left(q \tilde{D}_{4} / 2^{p}\right)^{256}, \Lambda_{2}=\left(q \tilde{D}_{4}\right)^{256}$ and $\Lambda_{3}=q \mathbb{Z}^{1024}$. We point out that a dither is not required in our algorithm like in [7, 8] since the modulus $q$ is an even number. Unlike NewHope, the proposed protocol is based on Module-LWE and uses the same parameters $n, d, k$ as in CRYSTALS - Kyber $[10,11]$. In order to obtain 256 bits of key with $n=256$, a higher-dimensional lattice than $\tilde{D}_{4}$ is needed.

\section{ERROR PROBABILITY BOUNDS}

In this section, we provide more technical details on estimating the error probability $P_{e}=\mathbb{P}\{\mathbf{k} \neq \hat{\mathbf{k}}\}$. We will prove that in our described protocol, the parameter set we recommend in Table \yields $P_{e}<2^{-174}$.

\section{A. Reliability condition}

According to Section III the two keys $\mathbf{k}$ and $\hat{\mathbf{k}}$ would be identical whenever $Q_{\Lambda_{2}}(v-r) \bmod \Lambda_{3}$ and
$Q_{\Lambda_{2}}\left(v^{\prime}-r\right) \bmod \Lambda_{3}$ are equal. Setting $e_{Q}=v-Q_{\Lambda_{1}}(v)$ we can show that a sufficient condition is $Q_{\Lambda_{2}}\left(\left(v-v^{\prime}\right)+e_{Q}\right)=$ 0 ; or more appropriately:

$$
Q_{\frac{q}{2}\left(1-\frac{1}{2^{p-1}}\right) E_{8}}\left(v-v^{\prime}\right)=0
$$

because $e_{Q} \in \mathcal{V}\left(\Lambda_{1}\right)$. For clearer notations, we assign $C:=$ $\frac{q}{2}\left(1-\frac{1}{2^{p-1}}\right)$. Let $\omega$ denote the error difference between $v$ and $v^{\prime}$ for which

$$
\begin{aligned}
v-v^{\prime} & =\mathbf{b} \cdot \mathbf{s}^{\prime}+e^{\prime \prime}-\mathbf{u} \cdot \mathbf{s} \\
& =(\mathbf{A} \mathbf{s}+\mathbf{e}) \cdot \mathbf{s}^{\prime}+e^{\prime \prime}-\left(\mathbf{A}^{T} \mathbf{s}^{\prime}+\mathbf{e}^{\prime}\right) \cdot \mathbf{s} \\
& =\mathbf{e} \cdot \mathbf{s}^{\prime}-\mathbf{e}^{\prime} \cdot \mathbf{s}+e^{\prime \prime} \in R_{q} \\
& =e_{1} s_{1}^{\prime}+\cdots+e_{d} s_{d}^{\prime}+-e_{1}^{\prime} s_{1}-\cdots-e_{d}^{\prime} s_{d}+e^{\prime \prime} \\
& =\omega_{1}+\omega_{2}+\ldots \omega_{d}+e^{\prime \prime}, \text { where } \omega_{i}=e_{i} s_{i}^{\prime}-e_{i}^{\prime} s_{i}
\end{aligned}
$$

This can be written in polynomial form as:

$$
\omega(X)=\omega_{1}(X)+\omega_{2}(X)+\ldots \omega_{d}(X)+e^{\prime \prime}(X)
$$

So for $\kappa=0, \ldots, L-1$, the expression of $\omega^{(\kappa)}(Y)$ will be:

$\sum_{j=1}^{d} \sum_{i=0}^{L-1} Y^{\delta_{i, \kappa}}\left[e_{j}{ }^{(i)} s_{j}^{\prime(\kappa-i)}(Y)-e_{j}^{\prime(i)} s_{j}^{(\kappa-i)}(Y)\right]+e^{\prime \prime(\kappa)}(Y)$.

As in [8, 21], we will consider a union bound over all Voronoirelevant vectors. Note that $\omega^{(\kappa)}(Y)$ is still a polynomial with 8 coefficients. Decoding will be correct if $\omega^{(\kappa)} \in C \cdot \mathcal{V}\left(E_{8}\right)$ for all $\kappa=0,1,2, \ldots, L-1$. More formally, $\omega^{(\kappa)} \in C$. $\mathcal{V}\left(E_{8}\right) \Longleftrightarrow\left\langle\omega^{(\kappa)}, v\right\rangle \leq \frac{\|v\|_{2}^{2}}{2}, \forall v \in C\left(\mathrm{VR}_{1} \cup \mathrm{VR}_{2}\right)$.

We mention that multiplying the vector form of $e^{(i)}(Y)$ by $Y$ is equivalent to a right shift with a minus sign on the first term. Using this and the fact that the distributions of $e_{j}^{(\kappa-i)}$ and $e_{j}^{\prime(\kappa-i)}$ are invariant by $\operatorname{conj}(\cdot)$ and by multiplication by -1 , as well as the distributions of $s_{j}{ }^{(i)}$ and $s_{j}^{\prime(i)}$ are also invariant by shifting and by multiplication by -1 , we obtain a more compact form of $\left\langle\omega^{(\kappa)}, v\right\rangle$ :

$$
\left\langle\omega^{(\kappa)}, v\right\rangle=\left\langle\left(\tilde{s}^{\prime}{ }_{j}, \tilde{s}_{j}\right)_{j=1, \ldots, d}, W_{v, \kappa}\right\rangle+\left\langle e^{\prime \prime(\kappa)}, v\right\rangle
$$

where $\tilde{s}_{j}$ and $\tilde{s}_{j}^{\prime}$ are $n$ dimensional vectors of independent centered binomial coefficients, and

$$
W_{v, \kappa}=C \cdot\left[\operatorname{conj}\left(e_{j}^{(\kappa-i)}\right) \cdot v, \ldots, \operatorname{conj}\left(e_{j}^{\prime(\kappa-i)}\right) \cdot v\right]_{\substack{i=0, \ldots, L-1, j=1, \ldots, d}}
$$

can be identified to $W_{v, \kappa}=C \cdot\left(e^{(0)} \cdot v, \ldots e^{(2 L \times d-1)} \cdot v\right)$, where each component $e^{(i)} \cdot v$ is a polynomial multiplication of an 8-dimensional vector $e^{(i)}$ by a Voronoi-relevant vector $v$. The multiplication is done modulo $\left(Y^{8}+1\right)$. Moreover, the $e^{(i)} \mathrm{s}$ are independent with centered binomial coefficients, distributed also independently. For instance, if $v_{1} \in \mathrm{VR}_{1}$ is a Voronoi-relevant vector of type 1 , then $W_{v_{1}, \kappa}$ is given by the general form

$$
\frac{C}{2} \cdot\left[\left( \pm e_{i_{0}}^{(0)} \pm e_{i_{1}}^{(0)}, \ldots, \pm e_{i_{6}}^{(0)} \pm e_{i_{7}}^{(0)}\right) ; \ldots\right] .
$$

However, if $v_{2} \in \mathrm{VR}_{2}$ is a Voronoi-relevant vector of type 2, then each component of $e^{(i)} \cdot v$ of $W_{v_{2}, \kappa}$ is of the form:

$$
\frac{C}{4}\left( \pm e_{i_{0}}^{(i)} \pm e_{i_{1}}^{(i)} \pm e_{i_{2}}^{(i)} \pm e_{i_{3}}^{(i)} \pm e_{i_{4}}^{(i)} \pm e_{i_{5}}^{(i)} \pm e_{i_{6}}^{(i)} \pm e_{i_{7}}^{(i)}\right) .
$$




\section{B. Error probability calculations}

Recall that an error occurs if $\omega^{(\kappa)} \notin C \cdot \mathcal{V}\left(E_{8}\right)$ for some $\kappa=0, \ldots, L-1$. So one can bound $P_{e}$ by

$$
\mathbb{P}\left\{\exists \kappa, \exists v \in C\left(\mathrm{VR}_{1} \cup \mathrm{VR}_{2}\right):\left\langle\omega^{(\kappa)}, v\right\rangle>\frac{\|v\|_{2}^{2}}{2}\right\}
$$

Using the fact that $\left\langle\omega^{(\kappa)}, v\right\rangle=\left\langle\left(\tilde{s^{\prime}}, \tilde{s}\right), W_{v, \kappa}\right\rangle+\left\langle e^{\prime \prime(\kappa)}, v\right\rangle$ we obtain:

$$
P_{e} \leq \sum_{\kappa=0}^{L-1} \mathbb{P}\left\{\exists v:\left\langle\left(\tilde{s^{\prime}}, \tilde{s}\right), W_{v, \kappa}\right\rangle>\frac{\|v\|_{2}^{2}}{2}-\left\langle e^{\prime \prime(\kappa)}, v\right\rangle\right\}
$$

Observing that $\frac{\|v\|_{2}^{2}}{2}-\left\langle e^{\prime \prime(\kappa)}, v\right\rangle \geq C^{2}-2 k C$ for type 1 vectors (resp. $C^{2}-4 k C$ for type 2 ), we can bound each term by computing the distribution of $\left\langle\left(s^{\prime}, s\right), W_{v, \kappa}\right\rangle$, which is a sum of 192 i.i.d. random variables of the form $e^{(i)} \cdot v$. Details are omitted due to lack of space. From our numerical simulations, we obtain the following table:

\begin{tabular}{|l|l|l|l|l|}
\hline Error Probability Bound & $p=2$ & $p=3$ & $p=4$ & $p=5$ \\
\hline$q=2^{11}, k=2$ & $2^{-48}$ & $2^{-113}$ & $2^{-153}$ & $2^{-174}$ \\
\hline$q=2^{12}, k=4$ & $2^{-47}$ & $2^{-112}$ & $2^{-152}$ & $2^{-172}$ \\
\hline$q=2^{13}, k=4$ & $2^{-193}$ & $2^{-390}$ & $2^{-499}$ & $2^{-557}$ \\
\hline
\end{tabular}

TABLE II

Upper bound for error probability for different values of moduli $q$, noise parameter $k$ and reconciliation rate parameter $p$

\section{IND-CPA SECURITY}

We will prove that, with the choice of $q$ in Section IV, the algorithm is IND-CPA secure, assuming the hardness of $M$-LWE given two samples. This proof is generic and holds in the setting of the key generation protocol in Section IIII independently of the choice of the lattices $\Lambda_{1}$ and $\Lambda_{2}$ as long as the CVP can be done efficiently. We follow the same argument as Section 4.2 in [7]. We consider the adjacent games below:

$$
\begin{aligned}
& \text { Game } 1 \\
& \mathbf{A} \stackrel{\$}{\leftarrow} R_{q}^{d \times d} \\
& (\mathbf{b}, \mathbf{s}) \leftarrow \operatorname{Gen}(\mathbf{A}) \\
& ((\mathbf{u}, r), \mathbf{k}) \leftarrow \operatorname{Encaps}(\mathbf{A}, \mathbf{b}) \\
& \text { Output }(\mathbf{A}, \mathbf{b},(\mathbf{u}, r), \mathbf{k}) \\
& \mathbf{A} \stackrel{\$}{\leftarrow} R_{q}^{d \times d} \\
& \mathbf{b} \stackrel{\$}{\leftarrow} R_{q}^{d} \\
& ((\mathbf{u}, r), \mathbf{k}) \leftarrow \operatorname{Encaps}(\mathbf{A}, \mathbf{b}) \\
& \operatorname{Output}(\mathbf{A}, \mathbf{b},(\mathbf{u}, r), \mathbf{k}) \\
& \begin{array}{c}
\text { Game } 1 \\
\mathbf{A} \stackrel{\$}{\leftarrow} R_{q}^{d \times d}
\end{array} \\
& (\mathbf{b}, \mathbf{s}) \leftarrow \operatorname{Gen}(\mathbf{A}) \\
& ((\mathbf{u}, r), \mathbf{k}) \leftarrow \operatorname{Encaps}(\mathbf{A}, \mathbf{b}) \\
& \mathbf{k}^{*} \stackrel{\$}{\leftarrow} \Lambda_{2} / \Lambda_{3} \\
& \text { Output }\left(\mathbf{A}, \mathbf{b},(\mathbf{u}, r), \mathbf{k}^{*}\right) \\
& (\mathbf{A}, \mathbf{b}) \stackrel{\$}{\leftarrow} R_{q}^{d \times d} \times R_{q}^{d} \\
& (\mathbf{u}, v) \stackrel{\$}{\leftarrow} R_{q}^{d} \times R_{q} \\
& r=\operatorname{HelpRec}(v) \\
& \mathbf{k}^{*} \stackrel{\$}{\leftarrow} \Lambda_{2} / \Lambda_{3} \\
& \text { Output }\left(\mathbf{A}, \mathbf{b},(\mathbf{u}, r), \mathbf{k}^{*}\right)
\end{aligned}
$$

Notice that Game 1 is the "real" game defined in Section [II and Game 1' is the "ideal" one. Our aim is to prove that Game 1 and Game 1' are computationally indistinguishable. We'll do so sequentially.

Clearly Game 1 and Game 2 are computationally indistinguishable under the assumption of hardness of $M$-LWE.

To prove that Game 2 and Game 3 are computationally indistinguishable, we use the following Theorem which is essentially a consequence of the Crypto Lemma [22, Lemma 4.1.1]. It guarantees uniformity of the key without a dither.

Theorem 1. If $v \in R_{q}$ is uniformly random, then $\mathbf{k}=$ $\operatorname{Rec}(v, r)$ is uniformly random, given $r=\operatorname{HelpRec}(v)$.

Proof: For fixed $\mathbf{k}, \mathbf{k}^{\prime} \in \Lambda_{2} / \Lambda_{3}$, we define $\forall v \in R_{q}$,

$$
\pi_{\mathbf{k}, \mathbf{k}^{\prime}}(v)=\left(v-\mathbf{k}+\mathbf{k}^{\prime}\right) \bmod \Lambda_{3} .
$$

Notice that $\pi_{\mathbf{k}, \mathbf{k}^{\prime}}(v) \in R_{q}$ because $\left(-\mathbf{k}+\mathbf{k}^{\prime}\right) \in \Lambda_{2} \subseteq \mathbb{Z}^{n}$ and hence $\pi_{\mathbf{k}, \mathbf{k}^{\prime}}(v) \in \mathbb{Z}_{q}^{n}$. So $\pi_{\mathbf{k}, \mathbf{k}^{\prime}}$ is a permutation of $R_{q}$ by Lemma1 The proof of Theorem 1 results from these lemmas:

Lemma 2. $\forall \mathbf{k}, \mathbf{k}^{\prime} \in \Lambda_{2} / \Lambda_{3}$ and $\forall v \in R_{q}$ we have $\operatorname{HelpRec}(v)=\operatorname{HelpRec}\left(\pi_{\mathbf{k}, \mathbf{k}^{\prime}}(v)\right)$.

Proof:

$$
\begin{aligned}
r^{\prime} & =Q_{\Lambda_{1}}\left(\pi_{\mathbf{k}, \mathbf{k}^{\prime}}(v)\right) \bmod \Lambda_{2} \\
& =Q_{\Lambda_{1}}\left(\left(v-\mathbf{k}+\mathbf{k}^{\prime}\right) \bmod \Lambda_{3}\right) \bmod \Lambda_{2} \\
& =Q_{\Lambda_{1}}\left(v-\mathbf{k}+\mathbf{k}^{\prime}-Q_{\Lambda_{3}}\left(v-\mathbf{k}+\mathbf{k}^{\prime}\right)\right) \bmod \Lambda_{2} \\
& =\left(Q_{\Lambda_{1}}(v)-\mathbf{k}+\mathbf{k}^{\prime}-Q_{\Lambda_{3}}\left(v-\mathbf{k}+\mathbf{k}^{\prime}\right)\right) \bmod \Lambda_{2} \\
& =Q_{\Lambda_{1}}(v) \bmod \Lambda_{2}=r .
\end{aligned}
$$

Lemma 3. Suppose that $\mathbf{k}=\operatorname{Rec}(v, r)=Q_{\Lambda_{2}}(v-r)$ $\bmod \Lambda_{3}$. Then $\forall \mathbf{k}^{\prime} \in \Lambda_{2} / \Lambda_{3}$ we have $\mathbf{k}^{\prime}=\operatorname{Rec}\left(\pi_{\mathbf{k}, \mathbf{k}^{\prime}}(v), r\right)$.

Proof:

$$
\begin{aligned}
\operatorname{Rec}\left(\pi_{\mathbf{k}, \mathbf{k}^{\prime}}(v), r\right) & =\left[Q_{\Lambda_{2}}(v-r)-\mathbf{k}+\mathbf{k}^{\prime}\right] \bmod \Lambda_{3} \\
& =\left[Q_{\Lambda_{2}}(v-r) \bmod \Lambda_{3}-\mathbf{k}+\mathbf{k}^{\prime}\right] \bmod \Lambda_{3} \\
& =\left[\mathbf{k}-\mathbf{k}+\mathbf{k}^{\prime}\right] \bmod \Lambda_{3}=\mathbf{k}^{\prime}
\end{aligned}
$$

Corollary 1. $\forall \mathbf{k}, \mathbf{k}^{\prime} \in \Lambda_{2} / \Lambda_{3}$ and $\forall v \in R_{q}$, there exist $v^{\prime}=\pi_{\mathbf{k}, \mathbf{k}^{\prime}}(v)$ such that $\operatorname{HelpRec}(v)=\operatorname{HelpRec}\left(\pi_{\mathbf{k}, \mathbf{k}^{\prime}}(v)\right)$, and $\mathbf{k}=\operatorname{Rec}(v, r) \Longleftrightarrow \mathbf{k}^{\prime}=\operatorname{Rec}\left(\pi_{\mathbf{k}, \mathbf{k}^{\prime}}(v), r\right)$.

We conclude the proof of Theorem 1 by showing that $\mathbf{k}$ is uniform and independent of $r$ when $v$ is uniform:

$$
\begin{aligned}
\mathbb{P}\{\mathbf{k} \mid r\} & =\sum_{v \in R_{q}} \mathbb{P}\{v\} \cdot \mathbb{P}\{\mathbf{k} \mid r, v\} \\
& =\sum_{v \in R_{q}} \mathbb{1}_{\left\{\begin{array}{c}
r=\operatorname{HelpRec}(v) \\
\mathbf{k}=\operatorname{Rec}(v, r)
\end{array}\right\}} \cdot \mathbb{P}\{v\} \\
& =\sum_{v \in R_{q}} \mathbb{1}_{\left\{\begin{array}{c}
r=\operatorname{HelpRec}\left(\pi_{\mathbf{k}, \mathbf{k}^{\prime}}(v)\right) \\
\mathbf{k}^{\prime}=\operatorname{Rec}\left(\pi_{\mathbf{k}, \mathbf{k}^{\prime}}(v), r\right)
\end{array}\right\}} \cdot \mathbb{P}\{v\} \\
& =\sum_{v^{\prime} \in R_{q}} \mathbb{1}_{\left\{\begin{array}{l}
r=\operatorname{HelpRec}\left(v^{\prime}\right) \\
\mathbf{k}^{\prime}=\operatorname{Rec}\left(v^{\prime}, r\right)
\end{array}\right\}} \cdot \mathbb{P}\left\{v^{\prime}\right\} \\
& =\sum_{v^{\prime} \in R_{q}} \mathbb{P}\left\{v^{\prime}\right\} \cdot \mathbb{P}\left\{\mathbf{k}^{\prime} \mid r, v^{\prime}\right\}=\mathbb{P}\left\{\mathbf{k}^{\prime} \mid r\right\} .
\end{aligned}
$$

Returning to Game 2 and Game 3, we construct an efficient reduction $\mathcal{S}$ as follows: it takes as input two pairs $(\mathbf{A}, \mathbf{u}),(\mathbf{b}, v)$, and outputs

$$
(\mathbf{A}, \mathbf{b},(\mathbf{u}, r=\operatorname{HelpRec}(v)), \mathbf{k}=\operatorname{Rec}(v, r)) .
$$


After that, we will take two indistinguishable inputs, and hence, by efficiency of $\mathcal{S}$, get two indistinguishable outputs. First suppose that the inputs are $M$-LWE instances; i.e. $\mathbf{u}=$ $\mathbf{A}^{T} \mathbf{s}^{\prime}+\mathbf{e}^{\prime}$ and $v=\mathbf{b} \cdot \mathbf{s}^{\prime}+e^{\prime \prime}$. So $\mathbf{A}$ must be uniformly random, and $\mathbf{b}$ is indistinguishable from uniform. Hence, the output of $\mathcal{S}$ will be exactly as in Game 2 . Now suppose that the inputs given to $\mathcal{S}$ are uniformly random in and independent, then the outputs of $\mathcal{S}$ are exactly as in Game 3. In fact, $\mathbf{A}, \mathbf{b}, \mathbf{u}, v$ are uniform, and hence by Theorem 1, $\mathbf{k}$ is uniformly random conditioned on $r=\operatorname{HelpRec}(v)$.

To show that Game 3 and Game 1' are indistinguishable, we modify Game 1 and Game 2 by choosing $\mathrm{k}^{*} \stackrel{\$}{\leftarrow} \Lambda_{2} / \Lambda_{3}$ and output it instead of $\mathbf{k}$. In this case Game 1 becomes Game 1'. Let Game 2' be the modified version of Game 2. By the same reasoning as above, we can prove that Game 1' is computationally indistinguishable from Game 2' and Game 3.

Remark 2. Following the steps in [7, Section 5], we can construct a passively secure encryption scheme based on our passively secure KEM, which yields an actively secure encryption scheme and an actively secure key transport protocol.

\section{SECURITY AGAINST KNOWN ATTACKS}

We study the hardness of Module-LWE by considering it as an LWE problem, since, to date, the best known attacks don't make use of the module structure. There are numerous attacks to consider, however, we essentially deal with two BKZ attacks, referred to as primal and dual attacks (see [8, 15, 23] for details). The cost of the primal attack and dual attack are given in Table III using NewHope's script 2 to do the calculations. We also make a comparison between our protocol and [10, 11, 15]'s in the term of security, and obtain a significantly improved security with respect to [10, 11] with smaller decryption error rate, and the same level of security with better modulus and smaller error rate comparing to [15].

\begin{tabular}{|l||l|l||lcc|}
\hline Attack & $m$ & $b$ & $\begin{array}{l}\text { Known } \\
\text { Classical }\end{array}$ & $\begin{array}{l}\text { Known } \\
\text { Quantum }\end{array}$ & $\begin{array}{l}\text { Best } \\
\text { Plausible }\end{array}$ \\
\hline \hline Saber-KEM: $q=2^{13}=8192, n=256, k=8, d=3, P_{e} \leq 2^{-136}$ \\
\hline Primal & 765 & 667 & 195 & 176 & 138 \\
\hline Dual & 765 & 664 & 194 & 176 & 137 \\
\hline \hline Kyber768 Round $1: q=7681, n=256, k=4, d=3, P_{e} \leq 2^{-142}$ \\
\hline Primal & 714 & 613 & 179 & 162 & 127 \\
\hline Dual & 733 & 610 & 178 & 161 & 126 \\
\hline Our Protocol: $q=2^{12}=4096, n=256, k=4, d=3, P_{e} \leq 2^{-152}$ \\
\hline Primal & 730 & 667 & 195 & 177 & 138 \\
\hline Dual & 727 & 664 & 194 & 176 & 137 \\
\hline \hline Kyber768 Round 3: $q=3329, n=256, k=2, d=3, P_{e} \leq 2^{-164}$ \\
\hline Primal & 658 & 623 & 182 & 165 & 129 \\
\hline Dual & 670 & 620 & 181 & 164 & 128 \\
\hline Our Protocol: $q=2^{11}=2048, n=256, k=2, d=3, P_{e} \leq 2^{-174}$ \\
\hline Primal & 658 & 665 & 194 & 176 & 138 \\
\hline Dual & 651 & 662 & 194 & 176 & 137 \\
\hline
\end{tabular}

TABLE III

Core hardness of our protocol and comparison with the state of the art. $b$ denotes the block dimension of BKZ, and $m$ the number of used samples. The given costs are the smallest ones for all possible choices of $m$ and $b$.

${ }^{2}$ https://github.com/newhopecrypto/newhope/blob/master/scripts/PQsecurity.py

\section{ACKNOWLEDGMENTS}

The work of C. Saliba and L. Luzzi is supported by the INEX Paris-Seine AAP 2017. The authors would like to thank J.-P. Tillich for helpful comments.

\section{REFERENCES}

[1] R. L. Rivest, A. Shamir, and L. Adleman, "A method for obtaining digital signatures and public-key cryptosystems," Communications of the ACM, vol. 21, no. 2, pp. 120-126, 1978 .

[2] W. Diffie and M. Hellman, "New directions in cryptography," IEEE transactions on Information Theory, vol. 22, no. 6, pp. 644-654, 1976.

[3] O. Regev, "On lattices, learning with errors, random linear codes, and cryptography," Journal of the ACM (JACM), vol. 56, no. 6, p. 34, 2009.

[4] C. Peikert and B. Waters, "Lossy trapdoor functions and their applications," SIAM Journal on Computing, no. 6, pp. 1803-1844, 2011.

[5] V. Lyubashevsky, C. Peikert, and O. Regev, "On ideal lattices and learning with errors over rings," in Annual International Conference on the Theory and Applications of Cryptographic Techniques. Springer, 2010, pp. 1-23.

[6] A. Langlois and D. Stehlé, "Worst-case to average-case reductions for module lattices," Designs, Codes and Cryptography, vol. 75, no. 3, pp. 565-599, 2015.

[7] C. Peikert, "Lattice cryptography for the internet," in International Workshop on Post-Quantum Cryptography. Springer, 2014, pp. 197219.

[8] E. Alkim, L. Ducas, T. Pöppelmann, and P. Schwabe, "Post-quantum key exchange-a new hope." in USENIX Security Symposium, 2016.

[9] E. Alkim, L. Ducas, T. Pöppelmann, and P. Schwabe, "NewHope without reconciliation." IACR Cryptology ePrint Archive, p. 1157, 2016.

[10] J. Bos, L. Ducas, E. Kiltz, T. Lepoint, V. Lyubashevsky, J. M. Schanck, P. Schwabe, G. Seiler, and D. Stehlé, "Crystals-Kyber: a CCA-secure module-lattice-based KEM," in 2018 IEEE European Symposium on Security and Privacy (EuroS\&P). IEEE, 2018, pp. 353-367.

[11] R. Avanzi, J. Bos, L. Ducas, E. Kiltz, T. Lepoint, V. Lyubashevsky, J. M. Schanck, P. Schwabe, G. Seiler, and D. Stehlé, "Crystals-Kyber algorithm specifications and supporting documentation," NIST PQC Round, 2020. [Online]. Available: https://pq-crystals.org/kyber/data/kyber-specification-round3.pdf

[12] J.-P. D'Anvers, Q. Guo, T. Johansson, A. Nilsson, F. Vercauteren, and I. Verbauwhede, "Decryption failure attacks on IND-CCA secure lattice-based schemes," in IACR International Workshop on Public Key Cryptography. Springer, 2019, pp. 565-598.

[13] T. Pöppelmann and T. Güneysu, "Towards practical lattice-based publickey encryption on reconfigurable hardware," in International Conference on Selected Areas in Cryptography. Springer, 2013, pp. 68-85.

[14] C. Peikert, O. Regev, and N. Stephens-Davidowitz, "Pseudorandomness of ring-LWE for any ring and modulus," in Proc. of the 49th Annual ACM SIGACT Symposium on Theory of Computing, 2017, pp. 461-473.

[15] J.-P. D'Anvers, A. Karmakar, S. S. Roy, and F. Vercauteren, "Saber: Module-LWR based key exchange, CPA-secure encryption and CCAsecure KEM," in International Conference on Cryptology in Africa. Springer, 2018, pp. 282-305.

[16] X. Lu, Y. Liu, Z. Zhang, D. Jia, H. Xue, J. He, B. Li, K. Wang, Z. Liu, and $\mathrm{H}$. Yang, "LAC: Practical ring-LWE based public-key encryption with byte-level modulus." IACR Cryptol. ePrint Arch., p. 1009, 2018.

[17] C. Peikert and Z. Pepin, "Algebraically structured LWE, revisited," in Theory of Cryptography Conference. Springer, 2019, pp. 1-23.

[18] J. H. Conway and N. J. A. Sloane, Sphere packings, lattices and groups. Springer Science \& Business Media, 2013, vol. 290.

[19] J. Convay and N. Sloane, "Fast quantizing and decoding algorithms for lattice quantizers," IEEE Trans Inform Theory, vol. 28, no. 2, pp. 227232, 1982.

[20] M. Song, S. Lee, D.-J. Shin, E. Lee, Y.-S. Kim, and J.-S. No, "Analysis of error dependencies on NewHope," IEEE Access, vol. 8, pp. 45443 45456,2020

[21] A. van Poppelen, "Cryptographic decoding of the Leech lattice," Master's thesis, Utrecht University, 2016

[22] R. Zamir, Lattice Coding for Signals and Networks: A Structured Coding Approach to Quantization, Modulation, and Multiuser Information Theory. Cambridge University Press, 2014.

[23] M. R. Albrecht, R. Player, and S. Scott, "On the concrete hardness of learning with errors," Journal of Mathematical Cryptology, vol. 9, no. 3, pp. 169-203, 2015. 